\title{
Pulmonary artery remodeling in transposition of the great arteries: Relevance for neoaortic root dilatation
}

\author{
Shirin Lalezari, MD ${ }^{\mathrm{a}, \mathrm{b}}$ \\ Mark G. Hazekamp, MD, $\mathrm{PhD}^{\mathrm{b}}$ \\ Margot M. Bartelings, MD, PhD \\ Paul H. Schoof, MD, PhD \\ Adriana C. Gittenberger-de Groot, $\mathrm{PhD}^{\mathrm{a}}$
}

From the Department of Anatomy and Embryology, Leiden University Medical Center, ${ }^{\mathrm{a}}$ and Department of Cardiothoracic Surgery, Leiden University Medical Center, ${ }^{\mathrm{b}}$ Leiden, The Netherlands.

Received for publication Sept 11, 2002; revisions requested Jan 6, 2003; revisions received March 18, 2003; accepted for publication April 11, 2003.

Address for reprints: A. C. Gittenberger-de Groot, $\mathrm{PhD}$, Department of Anatomy and Embryology, Leiden University Medical Center, Wassenaarseweg 62, PO Box 9602, 2300 AC Leiden, The Netherlands (E-mail: ACGitten@lumc.nl).

J Thorac Cardiovasc Surg 2003;126: 1053-60

Copyright (C) 2003 by The American Association for Thoracic Surgery

0022-5223/2003\$30.00+0

doi:10.1016/S0022-5223(03)00971-1
Objective: Transposition of the great arteries is currently treated by performing the arterial switch operation. Dilatation of the neoaortic root is a late complication with unknown cause. Samples of patients with untreated transposition of the great arteries and patients with normally related great arteries were compared to investigate a possible role for vascular remodeling in the dilatation process.

Methods: Aortic and pulmonary artery vessel wall and sinus samples were taken from 20 untreated human heart specimens with transposition of the great arteries and 9 age-matched, normal, postmortem human heart specimens, divided into 2 groups according to age. Routine histology and immunohistochemical staining for smooth muscle cell differentiation markers $\alpha$-smooth muscle actin, SM22, and calponin were performed.

Results: This study revealed structural differences between the normal aorta and pulmonary artery in the early group, which became more pronounced in the late group. In the early stage in transposition of the great arteries, no marked differences were seen between the aorta and pulmonary artery. With increasing age, however, there was a pronounced down-regulation of all smooth muscle cell markers in the pulmonary artery.

Conclusions: There is a structural difference between the normal neonatal aorta and pulmonary artery. The great arteries in transposition of the great arteries differ from each other and from normal vessels, indicating a structural vascular difference in transposition of the great arteries. In the pulmonary artery and sinus of untreated transposition of the great arteries, there is a dedifferentiation of smooth muscle cells with increasing age that we could not correlate to altered flow. This structural abnormality might provide an explanation for the neoaortic root dilatation that has been reported as a late complication of the arterial switch operation.

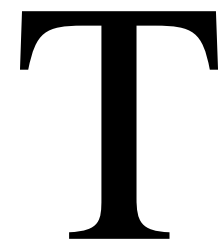

ransposition of the great arteries (TGA) is a heart defect that is found in approximately $5 \%$ of all newborns with congenital heart malformations. ${ }^{1}$ Currently, the operation of choice for TGA is the arterial switch operation (ASO). The first ASO was performed by Jatene and colleagues $^{2}$ in 1975 . Currently, most children undergo the ASO in the neonatal period with excellent survival ${ }^{3}$; however, late complications are being reported. ${ }^{4-6}$ One of the complications that needs further investigation is the neoaortic root dilatation that occurs after the ASO and the neoaortic valve insufficiency that may be associated with this dilatation. ${ }^{7,8}$ With this operation, the anatomic pulmonary root stays in place and becomes the neoaortic root that is subsequently subjected to systemic pressures.

Vascular remodeling occurs as a consequence of altered hemodynamic circumstances. ${ }^{9,10}$ In vessels exposed to alterations in shear stress and pressure differences, 


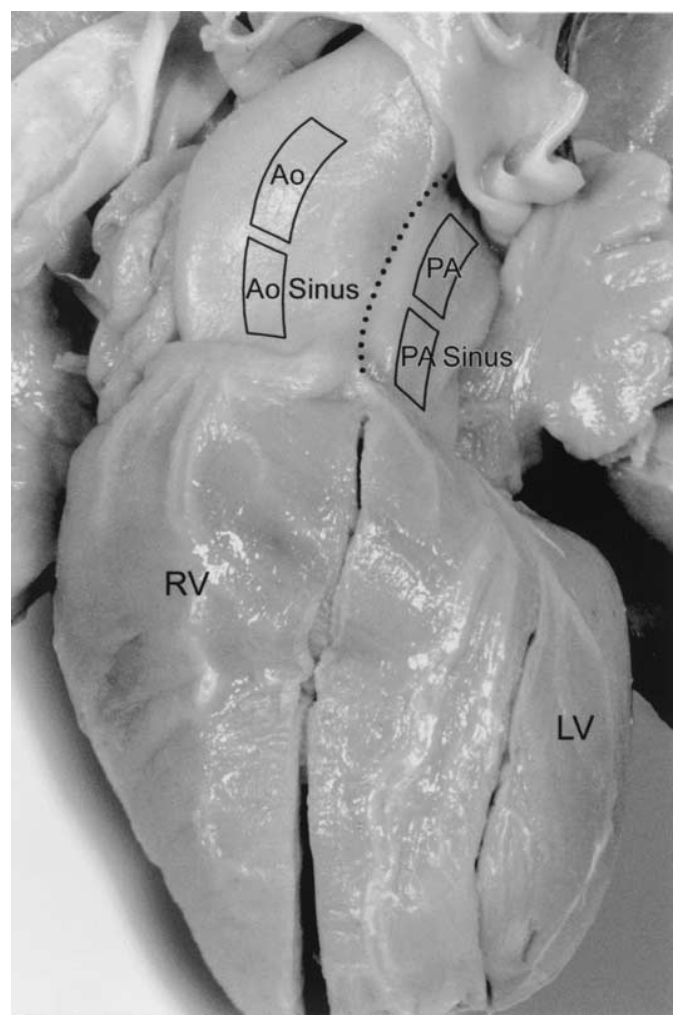

Figure 1. Example of a specimen with transposition of the great arteries with the squares representing the sample area. The samples in normal heart specimens were removed at the same location. Aortic (Ao) vessel wall; pulmonary artery (PA) vessel wall; aortic sinus (AoS); PA sinus (PAS).

vascular remodeling and the mechanisms involved have been described. ${ }^{11,12}$ Also, the behavior of the pulmonary autograft in the systemic circulation, as seen after the Ross procedure, has been examined in animal studies, showing an increase in smooth muscle cells (SMCs). ${ }^{13}$

To our knowledge, there are no reports on the histologic findings in the sinus and vessel wall of patients with TGA before or after surgical treatment. To elucidate the cause of late dilatation after ASO, we studied the histologic characteristics of the aorta and pulmonary artery (PA) of patients with untreated TGA and compared these findings with those of healthy individuals.

\section{Material and Methods}

\section{Tissue Samples}

Samples of the human aortic and pulmonary vessel wall were obtained from 20 postmortem, untreated heart specimens with TGA (from patients aged 1 day- 9 months) and 9 normal, postmortem heart specimens (from patients aged 1 day- 9 months) from the Leiden collection (Department of Anatomy and Embryology, Leiden University Medical Center, The Netherlands). Eleven heart specimens showed TGA with intact ventricular septum (IVS), whereas 9 other specimens showed TGA with ventricular septal
TABLE 1. Tissue specimens

\begin{tabular}{|c|c|c|}
\hline \multicolumn{3}{|c|}{ Normal hearts } \\
\hline \multicolumn{2}{|l|}{ No. } & Age \\
\hline \multicolumn{2}{|l|}{1} & $1 \mathrm{~d}$ \\
\hline \multicolumn{2}{|l|}{2} & $2 d$ \\
\hline \multicolumn{2}{|l|}{3} & $11 \mathrm{~d}$ \\
\hline \multicolumn{2}{|l|}{4} & $13 \mathrm{~d}$ \\
\hline \multicolumn{2}{|l|}{5} & $21 \mathrm{~d}$ \\
\hline \multicolumn{2}{|l|}{6} & 4 wk \\
\hline \multicolumn{2}{|l|}{7} & $10 w k$ \\
\hline \multicolumn{2}{|l|}{8} & $6 \mathrm{mo}$ \\
\hline \multicolumn{2}{|l|}{9} & $9 \mathrm{mo}$ \\
\hline \multicolumn{3}{|c|}{ Hearts with TGA } \\
\hline No. & Age & Diagnosis \\
\hline 1 & $1 \mathrm{~d}$ & $\mathrm{TGA}+\mathrm{PDA}$ \\
\hline 2 & $2 d$ & $\mathrm{TGA}+\mathrm{PDA}$ \\
\hline 3 & $4 d$ & $\mathrm{TGA}+\mathrm{BH}+\mathrm{PDA}$ \\
\hline 4 & $6 \mathrm{~d}$ & TGA + PDA \\
\hline 5 & $9 \mathrm{~d}$ & $\mathrm{TGA}+\mathrm{PDA}$ \\
\hline 6 & $12 \mathrm{~d}$ & $\mathrm{TGA}+\mathrm{BH}+\mathrm{PDA}$ \\
\hline 7 & $17 d$ & TGA + PDA \\
\hline 8 & $17 \mathrm{~d}$ & $T G A+V S D+P D A$ \\
\hline 9 & $22 \mathrm{~d}$ & TGA + PDA \\
\hline 10 & $1 \mathrm{mo}$ & $\mathrm{TGA}+\mathrm{VSD}+\mathrm{PDA}$ \\
\hline 11 & $2 \mathrm{mo}$ & $\mathrm{TGA}+\mathrm{VSD}$ \\
\hline 12 & $2 \mathrm{mo}$ & $\mathrm{TGA}+\mathrm{VSD}$ \\
\hline 13 & $3 \mathrm{mo}$ & $\mathrm{TGA}+\mathrm{BH}+\mathrm{PDA}$ \\
\hline 14 & $3 \mathrm{mo}$ & $\mathrm{TGA}+\mathrm{VSD}$ \\
\hline 15 & $4 \mathrm{mo}$ & $\mathrm{TGA}+\mathrm{VSD}+\mathrm{BH}$ \\
\hline 16 & $5 \mathrm{mo}$ & TGA + VSD \\
\hline 17 & $6 \mathrm{mo}$ & $\mathrm{TGA}+\mathrm{VSD}+\mathrm{BH}$ \\
\hline 18 & $7 \mathrm{mo}$ & $\mathrm{TGA}+\mathrm{BH}$ \\
\hline 19 & $8 \mathrm{mo}$ & TGA + VSD \\
\hline 20 & $9 \mathrm{mo}$ & $\mathrm{TGA}+\mathrm{PDA}$ \\
\hline
\end{tabular}

VSD, Ventricular septal defect; $B H$, Blalock-Hanlon septectomy; PDA, patent ductus arteriosus; TGA, transposition of the great arteries.

defect (VSD) (Figure 1, Table 1). From the same specimens, samples of aortic and pulmonary sinus were obtained. The sinus samples of the aorta were taken from the noncoronary sinus. The procedures were followed in accordance with institutional guidelines.

None of the control patients died of a cardiac or vessel-related cause. According to clinical reports, all patients with TGA died as the result of cardiac failure. We distinguished an early group (patients aged 1 day- 3 months) and a late group (patients aged 4-9 months). As mentioned in the clinical reports, a BlalockHanlon septectomy had been performed in 6 patients with TGA, and a morphologically patent ductus arteriosus was described in 12 patients (Table 1).

The specimens were fixed in ethanol and glycerin. The pieces of aortic and pulmonary vessel wall, as well as the sinus, were then routinely processed for light microscopy. Transverse sections (5 $\mu \mathrm{m})$ were mounted serially onto glycerin-coated glass slides and Starfrost glass slides (Klinipath BV, Duiven, The Netherlands). The paraffin-embedded tissue sections were deparaffinated, after which they were stained with hematoxylin-eosin, resorcin-fuchsin, 
TABLE 2. Routine histology of normal and TGA specimens

\begin{tabular}{|c|c|c|c|c|}
\hline \multicolumn{5}{|c|}{ Specimen number } \\
\hline \multirow{3}{*}{$\begin{array}{l}\text { Normal } \\
\text { hearts }\end{array}$} & \multicolumn{2}{|c|}{$H \& E$} & \multicolumn{2}{|c|}{ RF } \\
\hline & \multicolumn{2}{|c|}{$\begin{array}{c}\text { Organization } \\
\text { nucleus pattern: } \\
\text { compactness }\end{array}$} & \multicolumn{2}{|c|}{$\begin{array}{c}\text { Fragmentation elastic } \\
\text { laminae }\end{array}$} \\
\hline & Aorta & PA & Aorta & PA \\
\hline \multicolumn{5}{|c|}{ Early group } \\
\hline 1 & ++ & $\mathrm{N}$ & + & ++ \\
\hline 2 & ++ & + & + & ++ \\
\hline 3 & ++ & \pm & ++ & + \\
\hline 4 & +++ & $\overline{+}$ & + & ++ \\
\hline 5 & ++ & \pm & ++ & ++ \\
\hline 6 & +++ & ++ & + & ++ \\
\hline \multicolumn{5}{|c|}{ Late group } \\
\hline 7 & ++++ & \pm & + & +++ \\
\hline 8 & +++ & \pm & + & ++++ \\
\hline 9 & ++++ & + & ++ & +++ \\
\hline \multicolumn{5}{|c|}{ Hearts with TGA } \\
\hline \multicolumn{5}{|c|}{ Early group } \\
\hline 1 & \pm & \pm & ++ & ++ \\
\hline 2 & + & \pm & \pm & + \\
\hline 3 & - & - & +++ & +++ \\
\hline 4 & $\mathrm{~N}$ & $\mathrm{~N}$ & +++ & ++ \\
\hline 5 & - & \pm & +++ & + \\
\hline 6 & - & \pm & ++ & \pm \\
\hline 7 & $\mathrm{~N}$ & $\mathrm{~N}$ & ++ & - \\
\hline 8 & ++ & - & \pm & ++ \\
\hline 9 & $\mathrm{~N}$ & - & +++ & ++ \\
\hline 10 & - & + & \pm & ++ \\
\hline 11 & - & - & + & ++ \\
\hline 12 & - & + & \pm & ++ \\
\hline 13 & \pm & - & \pm & - \\
\hline 14 & - & - & \pm & +++ \\
\hline \multicolumn{5}{|c|}{ Late group } \\
\hline 15 & \pm & + & \pm & \pm \\
\hline 16 & + & \pm & ++ & + \\
\hline 17 & \pm & - & +++ & ++ \\
\hline 18 & - & - & + & ++ \\
\hline 19 & - & - & + & + \\
\hline 20 & + & \pm & \pm & + \\
\hline
\end{tabular}

$H \& E$, Hematoxylin-eosin; $R F$, resorcin-fuchsin; $P A$, pulmonary artery; $N$, not judgeable. ++++ : extreme; +++ : very; ++ : more than average; + : average; \pm : less than average; - : very little.

and modified van Gieson to study the morphology of the vessel walls.

\section{Immunohistochemistry}

For the identification of SMCs in the vessel wall and sinuses, the antibodies anti- $\alpha$-SM actin (1A4, Sigma, St Louis, Mo), antiSM22 ${ }^{14,15}$ (provided by Dr S. Sartore, Padova, Italy), and antihuman calponin ${ }^{16}$ (Sigma) were applied.

The sections were deparaffinated, followed by treatment with $0.3 \% \mathrm{H}_{2} \mathrm{O}_{2}$ in phosphate-buffered saline (PBS) $(\mathrm{pH} 7.3$ ) for 15 minutes to extinguish endogenous peroxidase activity. After this, sections were rinsed briefly in PBS twice and then in PBS with $0.05 \%$ Tween-20. Immunohistochemical staining was performed
TABLE 3. Immunohistochemistry of normal and TGA specimens

\begin{tabular}{|c|c|c|c|}
\hline \multicolumn{4}{|c|}{ Normal hearts (sinus and vessel wall) } \\
\hline \multirow{4}{*}{$\begin{array}{l}\text { Specimen } \\
\text { number }\end{array}$} & & $\alpha-$ & \\
\hline & IA4 & SM22 & Calponin \\
\hline & $\begin{array}{c}\text { Actin-positive SMCs } \\
\text { media }\end{array}$ & $\begin{array}{l}\text { Positive } \\
\text { SMCs }\end{array}$ & $\begin{array}{l}\text { Positive } \\
\text { SMCs }\end{array}$ \\
\hline & Aorta & PA & PA \\
\hline
\end{tabular}

\begin{tabular}{lcccc} 
number & Aorta & PA & PA & PA \\
\hline Early group & & & & \\
1 & ++ & + & + & + \\
2 & ++ & + & + & + \\
3 & ++ & + & \pm & + \\
4 & + & + & + & + \\
5 & ++ & + & + & + \\
6 & ++ & ++ & ++ & ++ \\
Late group & & & & \\
7 & ++ & ++ & ++ & ++ \\
8 & ++ & + & + & ++ \\
9 & + & ++ & ++ & + \\
Hearts TGA with (sinus and vessel wall) & &
\end{tabular}

\begin{tabular}{ccccc}
\hline Early group & & & & \\
1 & ++ & \pm & \pm & \pm \\
2 & ++ & \pm & \pm & \pm \\
3 & ++ & \pm & \pm & \pm \\
5 & ++ & \pm & -- & -- \\
6 & ++ & \pm & -- & \pm \\
8 & ++ & \pm & \pm & \pm \\
10 & ++ & - & --- & -- \\
11 & ++ & \pm & \pm & \pm \\
Late group & & & & \\
15 & ++ & -- & --- & --- \\
16 & + & \pm & -- & \pm \\
17 & ++ & \pm & -- & -- \\
18 & ++ & --- & --- & --- \\
19 & ++ & -- & --- & --- \\
20 & + & -- & --- & --
\end{tabular}

$1 A 4$, Anti- $\alpha$-smooth muscle actin; SMCs, smooth muscle cells; $P A$, pulmonary artery. ++ : more than average; + : average; \pm : less than average; - : little; - -: very little; - - : extremely little. All values: PA compared with aorta of same specimen. Specimens $4,7,9$, and 12 to 14 were not judgeable in the TGA group.

using 3-hour incubation with the primary antibodies diluted in PBS with $0.05 \%$ Tween-20 and $1 \%$ bovine serum albumin (Sigma) (1A4 1:10,000; anti-SM22 1:100; anti-human calponin 1:10,000).

Bound antibodies were detected using 1-hour incubation with horseradish peroxidase-conjugated rabbit anti-mouse antibody or horseradish peroxidase-conjugated swine anti-rabbit antibody (dilution 1:200, Dako A/S, Glostrup, Denmark), depending on whether the primary antibody was monoclonal or polyclonal. Control stainings were performed using PBS-Tween $0.05 \%$ and bovine serum albumin $1 \%$ as primary antibody.

The sections were then exposed to $0.04 \%$ diaminobenzidine tetrahydrochloride in $0.05 \mathrm{~mol} / \mathrm{L}$ Tris-maleate buffer $(\mathrm{pH} 7.6)$ with $0.006 \% \mathrm{H}_{2} \mathrm{O}_{2}$ for 10 minutes. After rinsing, the sections were counterstained with Mayer's hematoxylin for 7 seconds, dehydrated, and mounted in Entellan (Merck, Darmstadt, Germany). 

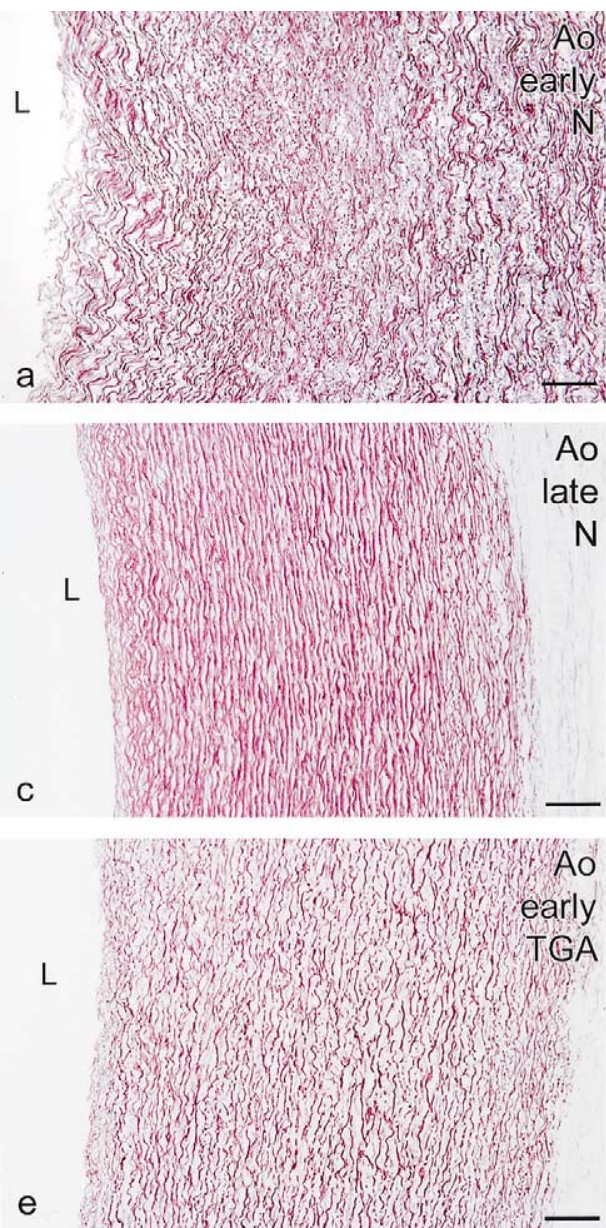

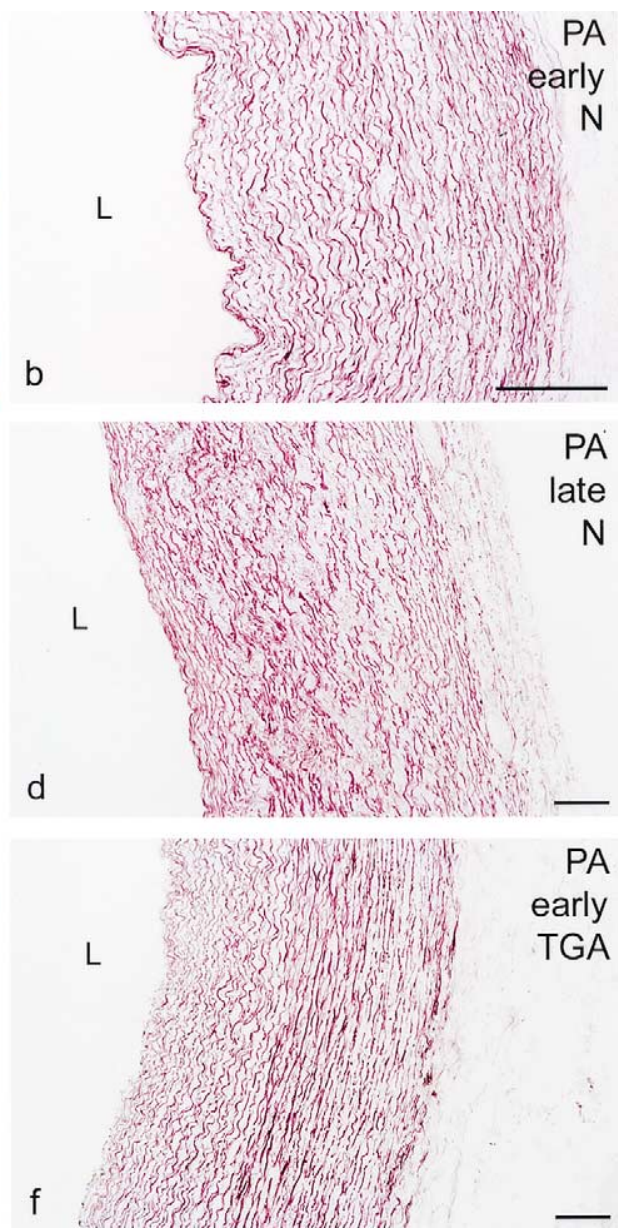

Figure 2. Resorcin-fuchsin staining of the vessel wall in normal hearts and hearts with TGA. a, b, Case 3; c, d, case 9; e, f, case 14 (Table 2). $N$, Normal heart; $L$, luminal side of the vessel.

The tissue sections stained with actin antibodies were then microscopically divided into sections of $1 \mathrm{~mm}^{2}$.

\section{Results}

\section{Routine Histology}

The histologic findings in both the vessel wall and sinus of normal hearts are summarized in Table 2. In the normal vessels stained with hematoxylin-eosin, a difference in cellular pattern between aorta and PA was distinguished in the early group. The cells in the aorta were more compactly organized compared with the PA, which became even more apparent in the late group. Also, the SMCs in the aortic media were mostly oriented longitudinally, whereas in the PA, this was not the case.

In regard to the elastic properties of the vessel wall in the early and late groups, the aortic elastic laminae were oriented in a densely organized, longitudinal fashion with increasing age as opposed to the laminae in the PA, which showed distinct fragmentation in a loosely organized, less dense structure (Figure 2).
In the hearts with TGA, routine histology did not reveal clear differences between the vessel wall and sinus of aorta and PA in the early and late groups. The orientation of the cells in both aorta and PA, stained with hematoxylin-eosin, appeared to be similar, in contrast with the findings in the normal vessels (Table 2, Figure 2). In general, the organization of cells was less compact compared with that in normal vessels.

\section{Immunohistochemistry}

The results are summarized in Table 3. In the normal hearts, both aorta and PA in the early and late groups showed actin-positive SMCs in the entire media of the vessel wall. In the PA, the staining pattern appeared more variable compared with the aorta (Figure 3, $a$ and $b$ ). This variation became more evident with increasing age (Figure 3, $e$ and $f$ ), which is supported by the findings in routine histology.

In TGA, actin-positive SMCs were also present in the complete media of both aorta and PA. No change in staining 


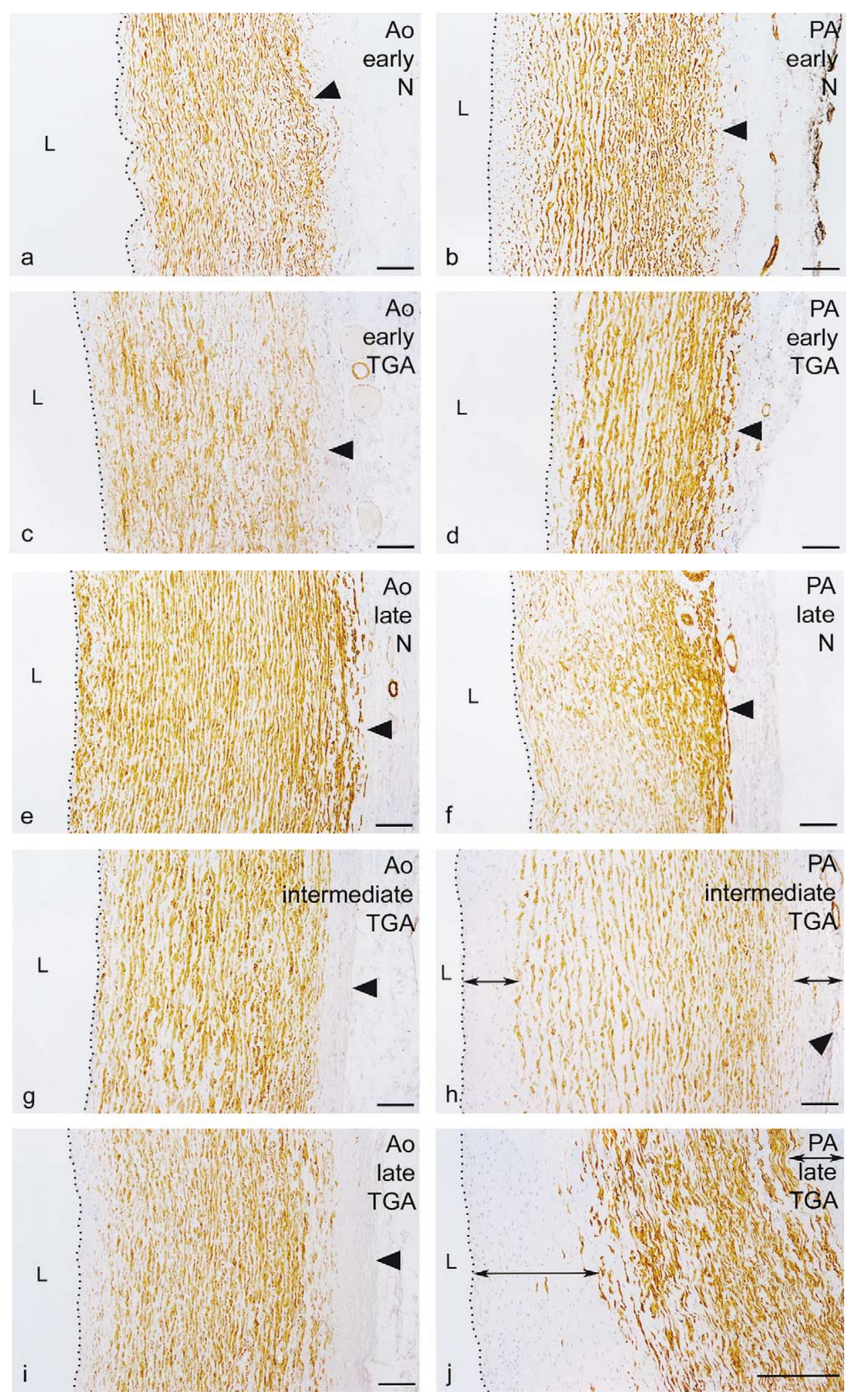

Figure 3. a-d, All figures showing anti- $\alpha$-smooth muscle actin (1A4) staining of the vessel wall of normal hearts and hearts with TGA in the early group. a, b, Case 1; c, d, case 8; e, j, 1A4 staining of the vessel wall of normal hearts and hearts with TGA in different age groups; e, f, case 9; $g$, h, case 15; i, j, case 18 (Table 3). $>$, Border between media and adventitia; $\leftrightarrow$, thickness of nonstained intima and media. 

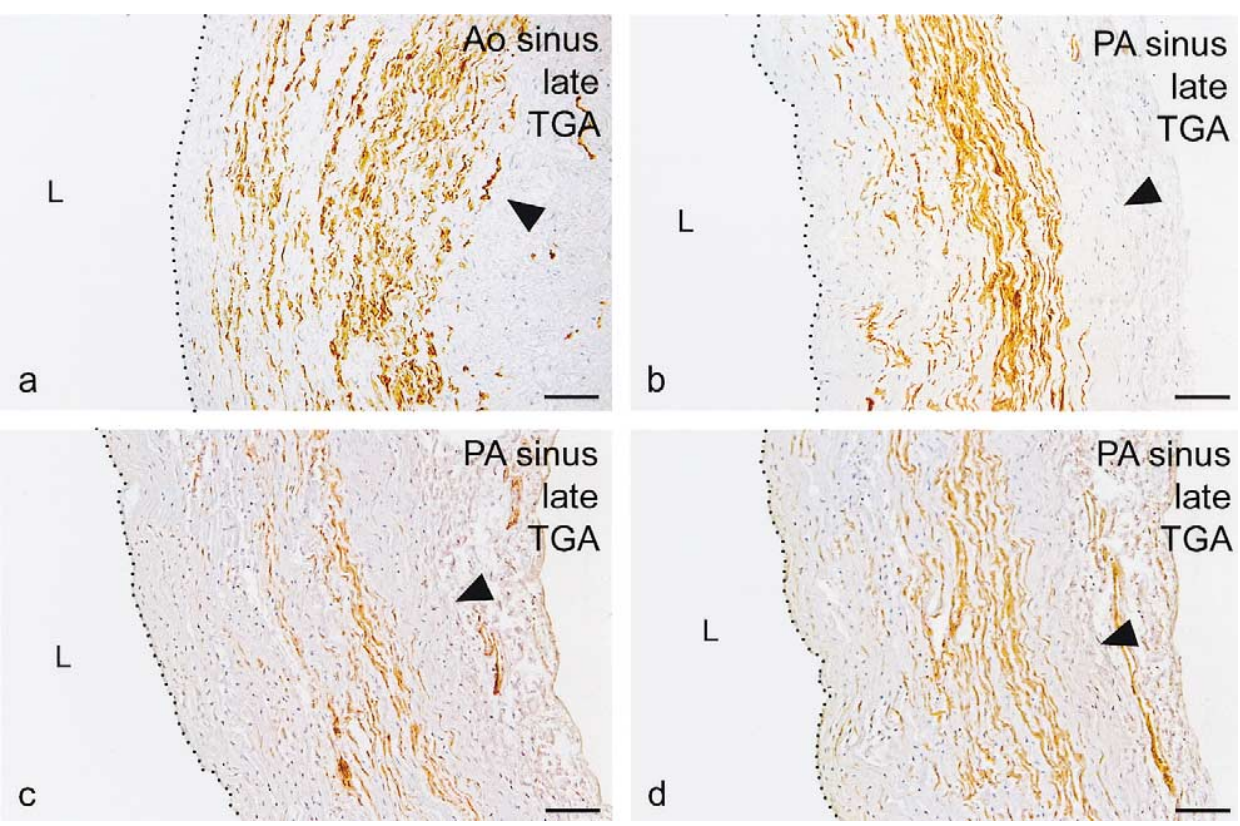

Figure 4. a, b, 1A4 staining of the aortic and PAS in the late TGA group (case 18, Table 3). c, The same PAS, stained with $\alpha$-SM22. d, The PAS stained with calponin.

pattern of the aorta was noted with increasing age (Figure 3, $c, g, i)$. The PA, however, did show evident changes. In the early group, the entire pulmonary media stained positive for 1A4 (Figure 3, d). With increasing age, a loss of actin positivity was seen in the inner and outer media (Figure 3, $h$ ) and was most marked in the inner media of the PA in the late group (Figure 3, $j$ ). The graphic relationship between age and area of negative actin staining in the media of aorta and PA in normal hearts and hearts with TGA is shown in Figure 5. Comparable results were obtained with the contractile SMC markers SM22 and calponin.

Study of the sinus wall of the aorta and PA in TGA showed results comparable to those found in the media of the vessel wall. We observed a marked loss of 1A4 expression in the PA sinus wall with increasing age, in comparison with the aortic sinus (Figure 4, $a-d$ ). These findings were again confirmed by SM22 and calponin expression that stayed within the $\alpha$-smooth muscle actin (1A4) boundaries.

The differences seen in the actin-stained sections of the vessel walls in the early and late groups are shown in Figure 5.

\section{Discussion}

The original aortic and pulmonary roots remain in situ after the ASO. Thus, the original aortic (neopulmonary) root remains connected to the right ventricle, whereas the original pulmonary (neoaortic) root remains attached to the left ventricle. One of the late complications of the ASO is dilatation of the neoaortic root with the possibility of (neo) aortic valve insufficiency. In this perspective, we studied the

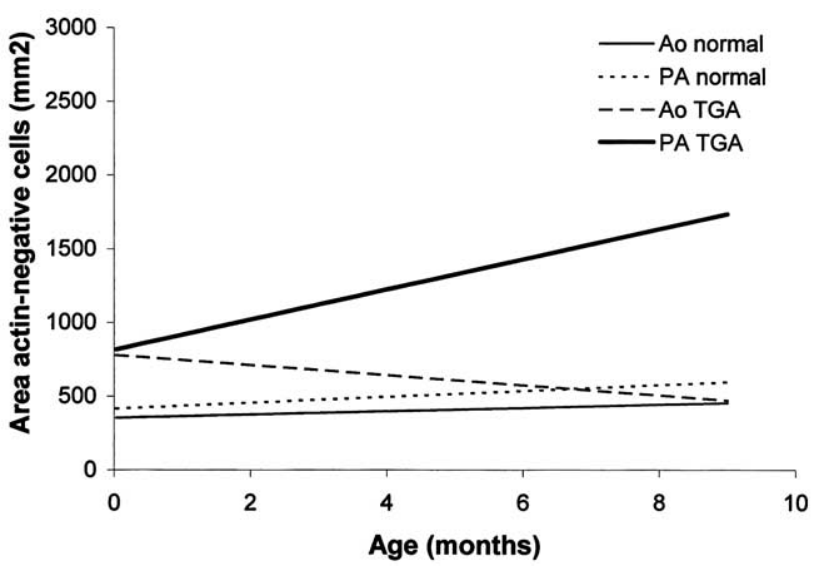

Figure 5. The trend between age and actin-negative area in normal aorta and PA vessel wall and sinuses, and in aorta and PA vessel wall and sinuses in TGA. The normal aorta and PA lose a small but not substantial amount of actin with increasing age. The aorta in TGA shows behavior more like normal vessels with increasing age. The PA in TGA shows a very clear trend in loss of actin-positive smooth muscle cells as the patient grows older.

structure of the great arteries and their roots in normal hearts and untreated hearts with TGA from patients aged 1 day to 9 months.

We had the unique opportunity to study the natural maturation of the great arteries and their roots in TGA, that is, in untreated, postmortem specimens from the preswitch era. The older patients with TGA in this series have lived for 
some time because of the existence of a VSD or a large ductus arteriosus together with the atrial septectomy that had been performed in the neonatal phase.

At birth, the structure of the aortic and pulmonary vessel walls and sinuses in normal hearts is different, and these differences become more evident with increasing age. No striking differences in structure were observed between the aorta and the PA of very young specimens with TGA, but a down-regulation of all SMC markers was observed in the PA of the older specimens with TGA. The expression of SMC markers ( $\alpha$-smooth muscle actin, SM22, and calponin) showed a significant decrease in the pulmonary vessel wall in TGA with increasing age.

Literature data indicate that, during development, the expression of SMC markers in the human aorta changes as the SMC phenotype develops. In the normal human aorta, SM actin accounts for approximately $80 \%$ of the fetal aortic media and increases to approximately $97 \%$ and $98 \%$ in the 6-month-old child and in the adult aortic media, respectively. ${ }^{17}$ No literature data are available on the SM-actin composition of the PA with increasing age.

In untreated TGA, the main vessels (ie, aorta and PA) are subjected to abnormal hemodynamic conditions. In both TGA with IVS and with VSD, pulmonary flow is increased. In TGA with VSD, both pulmonary flow and pressure are increased. In both conditions, pulmonary hypertension will develop rapidly.

Vascular remodeling as a response to changes in pressure has been reported; ${ }^{12,18}$ however, these reports are limited to the changes in vessel wall structure in the aorta or other smaller systemic arteries. Data on remodeling of the PA also exist. ${ }^{19}$ These reports deal with the changes occurring in the pulmonary vascular bed mainly as a response to pulmonary hypertension. ${ }^{20,21}$ Increased vessel wall thickness, elevated extracellular matrix proliferation, and vascular SMC hypertrophy or hyperplasia are described as the characteristic changes that take place in vascular remodeling as a response to elevated pressure, rather than dedifferentiation of pulmonary SMCs. ${ }^{22,23}$

After the Ross procedure, an increase in $\alpha$-SM actin, as well as an increase in the number of SMCs in the wall of the pulmonary autograft, has been reported. ${ }^{13}$

In the PA in TGA, we observed a decrease in the expression of SMCs with increasing age, whereas hypertrophy or an increase in the number of SMCs is observed in the pulmonary vessel wall after the Ross procedure and in pulmonary hypertension. Therefore, elevated pressure conditions are probably not the best way to explain this observation.

It is important to look into the role of blood flow in vessel wall remodeling in both TGA with IVS and with VSD, because pulmonary blood flow is increased in both conditions. Flow-mediated arterial remodeling has been de- scribed in animal models and humans. ${ }^{24,25}$ Buus and colleagues ${ }^{24}$ describe the changes in vessel wall structure in animal arteries exposed to high and low flow. In the lowflow model, phenotypic changes of SMCs were observed. Expression of the SMC differentiation marker 1A4 remained unchanged in both the high- and low-flow vessel walls, whereas calponin showed a significant decrease in the low-flow arteries, thus indicating dedifferentiation of SMCs with no change in proliferation activity of the SMCs compared with controls. In the high-flow arteries, dedifferentiation was also detected, using desmin mRNA as a differentiation marker, but this was not as significant as in the low-flow arteries and was most likely related to a higher proliferation activity.

In our study group, the PA vessel wall and sinus in TGA showed decreased expression of calponin. Notably, this decrease was also observed with 1A4 and SM22 in the PA samples of hearts with TGA with and without VSD. This implies that the observed remodeling of the SMCs in the "older" PA samples of hearts with TGA cannot be explained by flow-mediated mechanisms.

Schaper and colleagues ${ }^{26}$ describe dedifferentiation of vascular SMCs in growing collateral coronary arteries. This phenomenon is observed with SMC differentiation markers 1A4 and calponin. However, these dedifferentiated SMCs are primarily found in the neointima of the growing coronary collaterals. We investigated whether the SMC dedifferentiation that we observed in the "older" PA samples of hearts with TGA was specific for formation of a neointima. In our material, the phenomenon was found in both inner and outer media without changes in layering of the elastic laminae, whereas no intimal thickening occurred. This was also the case for the sinus region. Thus, the SMC downregulation in PA samples of hearts with TGA that we observed could not be explained by neointima formation.

To our best knowledge, this is the first report on structural differences of the great vessels between hearts with TGA and normal hearts. With the use of SMC differentiation markers, we were able to demonstrate a difference in SMC structure in the pulmonary wall and sinus between hearts with TGA and normal hearts, as well as a clear trend toward dedifferentiation of SMCs in the PA of "older" hearts with TGA. We could not explain this SMC downregulation by pressure- or flow-mediated mechanisms. Furthermore, the observation was not related to neointima formation. Thus, the findings in this study might imply that the PA in TGA is predetermined to develop in an essentially different way than the PA in a normal heart.

It could be speculated that early surgical repair (ASO) of TGA with or without VSD might reverse or decrease the reduction of SMCs in the PA vessel and sinus wall. However, further study will be needed to support this hypothesis. As far as we know, no data have been reported on a 
difference in late neoaortic root dilatation between ASOs performed early and late.

\section{Conclusion}

We found that the PA in untreated TGA showed a clear trend in reduction of actin-staining SMCs in the media throughout the first year of life. These histologic findings show that vascular remodeling occurs in the pulmonary vessel during the natural course of TGA. Increased flow or pressure conditions cannot explain these changes, thus indicating that the PA in TGA is structurally different compared with the PA in a normal heart. This observation may offer an explanation for the neoaortic dilatation that has been reported in the clinical follow-up of patients with TGA after the ASO ${ }^{8}$ Clinical observations that the vascular structure is different in patients with TGA, not only in the heart but also in other parts of the vascular system, have not been reported to date, but may have been overlooked.

\section{References}

1. Hoffman JI. Incidence of congenital heart disease: I. Postnatal incidence. Pediatr Cardiol. 1995;16:103-13.

2. Jatene AD, Fontes VF, Paulista PP, et al. Successful anatomic correction of transposition of the great vessels. A preliminary report. Arq Bras Cardiol. 1975;28:461-4.

3. Ptêtre R, Tamisier D, Bonhoeffer P, et al. Results of the arterial switch operation in neonates with transposition of the great arteries. Lancet. 2001;357:1826-30.

4. Williams WG, Quaegebeur JM, Kirklin JW, et al. Outflow obstruction after the arterial switch operation: a multiinstitutional study. J Thorac Cardiovasc Surg. 1997;114:975-90.

5. Kirklin JW, Blackstone EH, Tchervenkov CI, et al. Clinical outcomes after the arterial switch operation for transposition. Circulation. 1992; $86: 1501-15$.

6. Planche C, Lacour-Gayet F, Serraf A. Arterial switch. Pediatr Cardiol. 1998:19:297-307.

7. Hourihan M, Colan SD, Wernovsky G, et al. Growth of the aortic anastomosis, annulus and root after the arterial switch procedure performed in infancy. Circulation. 1993;88:615-20.

8. Hazekamp MG, Schoof PH, Suys BE, et al. Switch back: using the pulmonary autograft to replace the aortic valve after arterial switch operation. J Thorac Cardiovasc Surg. 1997;114:844-6.
9. Palmieri V, Bella JN, Arnett DK, et al. Aortic root dilatation at sinuses of valsalva and aortic regurgitation in hypertensive and normotensive subjects: The Hypertension Genetic Epidemiology Network Study. Hypertension. 2001;37:1229-35.

10. Raitakari OT, Celermajer DS. Flow-mediated dilatation. Br J Clin Pharmacol. 2000;50:397-404.

11. Owens GK. Role of mechanical strain in regulation of differentiation of vascular smooth muscle cells. Circ Res. 1996;79:1054-5.

12. Langille BL. Arterial remodeling: relation to hemodynamics. Can J Physiol Pharmacol. 1996;74:834-41.

13. Schoof PH, Gittenberger-de Groot AC, de Heer E, et al. Remodeling of the porcine pulmonary autograft wall in the aortic position. J Thorac Cardiovasc Surg. 2000;120:55-65.

14. Duband JL, Gimona M, Scatena M, et al. Calponin and SM22 as differentiation markers of smooth muscle: spatiotemporal distribution during avian development. Differentiation. 1993;55:1-11.

15. Li L, Miano JM, Cserjesi P, et al. SM22 $\alpha$, a marker of adult smooth muscle, is expressed in multiple myogenic lineages during embryogenesis. Circ Res. 1996;78:188-95.

16. Frid MG, Shekhonin BV, Koteliansky VE, et al. Phenotypic changes of human smooth muscle cells during development: late expression of heavy caldesmon and calponin. Dev Biol. 1992;153:185-93.

17. Glukhova MA, Frid MG, Koteliansky VE. Phenotypic changes of human aortic smooth muscle cells during development and in adult. $J$ Atheroscler Thromb. 1994;1(Suppl 1):S47-9.

18. Langille BL. Remodeling of developing and mature arteries: endothelium, smooth muscles, and matrix. J Cardiovasc Pharmacol. 1993; 21(Suppl 1):S11-7.

19. Stenmark KR, Mecham RP. Cellular and molecular mechanisms of pulmonary vascular remodeling. Ann Rev Physiol. 1997;59:89-144.

20. Tanaka Y, Schuster DP, Davis EC, et al. The role of vascular injury and hemodynamics in rat pulmonary artery remodeling. J Clin Invest. 1996;98:434-42.

21. Jeffery TK, Morrell NW. Molecular and cellular basis of pulmonary vascular remodeling in pulmonary hypertension. Prog Cardiovasc Dis. 2002;45:173-202.

22. Owens G, Schwartz S. Vascular smooth muscle cell hypertrophy and hyperploidy in the Goldblatt hypertensive rat. Circ Res. 1983;53:491501.

23. Owens G. Influence of blood pressure on development of aortic medial smooth muscle hypertrophy in spontaneously hypertensive rats. $\mathrm{Hy}$ pertension. 1987;9:178-87.

24. Buus CL, Pourageaud F, Fazzi GE, et al. Smooth muscle cell changes during flow-related remodeling of rat mesenteric resistance arteries. Circ Res. 2001;89:180-6.

25. Gibbons GH, Dzau VJ. The emerging concept of vascular remodeling. New Engl J Med. 1994;330:1431-38

26. Schaper W, Piek JJ, Munoz-Chapuli R, et al. Collateral circulation of the heart. In: Ware JA, Simons M, editors. Angiogenesis and cardiovascular disease. Oxford, New York: Oxford University Press; 1999. p. $159-98$ 